\title{
Sturzfolgen-Prophylaxe
}

\section{Ultraniedrigbett bietet Sicherheit und Freiheit}

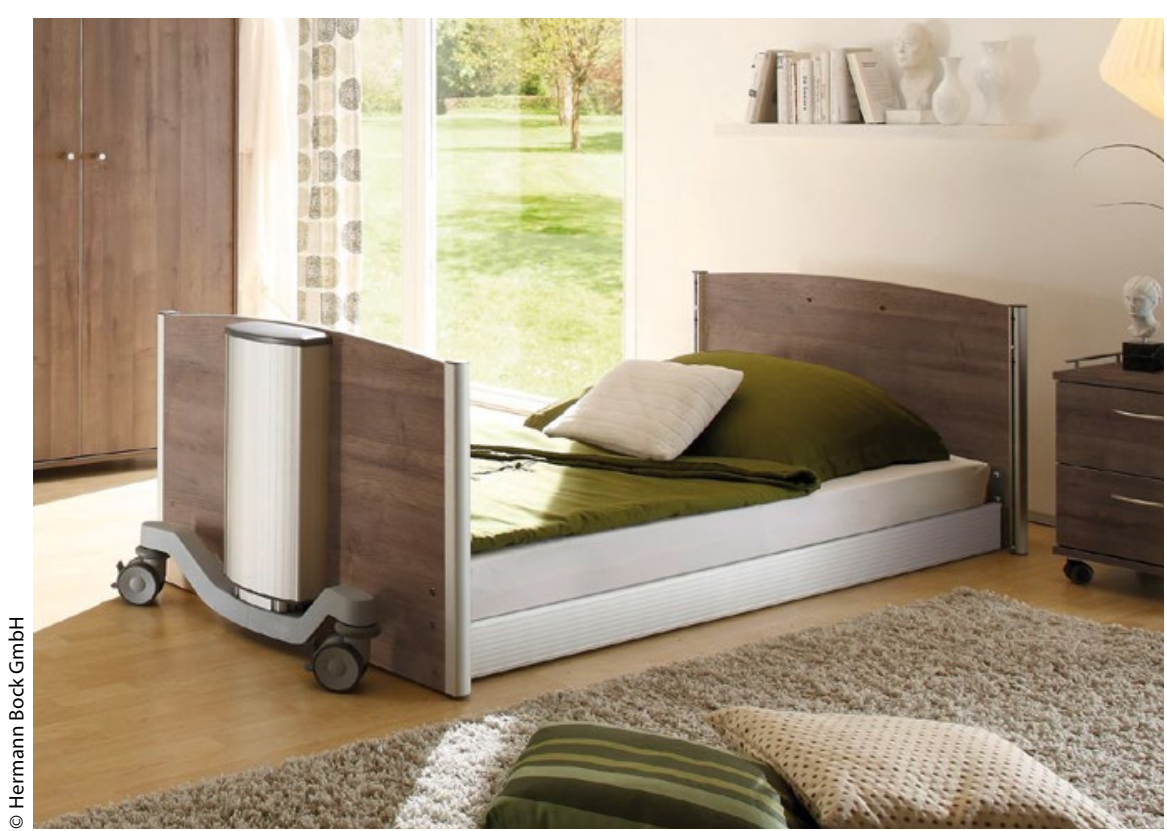

॥ Die Hermann Bock GmbH zeigte auf der Fachmesse Altenpflege 2017 in Nürnberg Innovationen für eine selbstbestimmte, mobilisierende Pflege. Unter anderem stellte der Hersteller das Pflegebett practico ultraniedrig 9,5/80 vor. Der Name ist Programm, denn das hoch funktionale Pflegebett lässt sich in dem kompletten Bereich zwischen 9,5 cm und $80 \mathrm{~cm}$ Pflegehöhe verfahren. Die extrem bodennahe
Ultraniedrigposition bietet Bewohnern und Pflegekräften den Vorteil, dass Verletzungen als Folge eines Herausgleitens aus dem Bett aus dieser geringen Höhe faktisch ausgeschlossen sind. Damit ermöglicht ein Ultraniedrigbett den Bewohnern Sicherheit bei absoluter Freiheit - ganz ohne Seitensicherungen oder sonstige einschränkenden Maßnahmen. Selbstverständlich ist das practico ultra- niedrig 9,5/80 mit voreingestellten Stops auch auf übliche Ein- und Ausstiegshöhen verfahrbar. „Wir haben bei unseren Neuentwicklungen stets auch die DNQP-Expertenstandards für die Pflege im Blick. Zum Beispiel bietet das practico ultraniedrig 9,5/80 im Sinne des Expertenstandards Mobilität mehr als nur Barrierefreiheit und Sicherheit. Die absolute Bodennähe ermöglicht vielen Patienten, nach einem unbeabsichtigten Herausgleiten eigenständig wieder ins Bett zu gelangen, zum Beispiel über den Vierfüsslerstand“, erläutert Martin Mühlenkord, Vertriebsleiter bei bock. Darüber hinaus bietet eine bodennahe Liegeposition insbesondere eingeschränkten Bewohnern auch ein haptisches Geborgenheits- und Sicherheitsgefühl, da die Bewohner aus dem Bett heraus den Boden ertasten können.

Für die Pflegekräfte bedeutet der Einsatz von Ultraniedrigbetten nicht nur Zeitersparnis durch den Verzicht auf das Auslegen von Abrollmatten oder sonstigen Maßnahmen zur Sturzfolgenprophylaxe. Durch die enorme Höhenverstellbarkeit ist das practico ultraniedrig 9,5/80 auch auf eine rückenschonende Pflegehöhe von bis $\mathrm{zu}$ $80 \mathrm{~cm}$ einstellbar.

www.bock.net

\section{Innovationstag Mittelstand}

\section{Neuheiten - Präsentation zur Erhöhung der Lebensqualität}

» Viele neue Entwicklungen aus dem Reha-Bereich, die den Alltag erleichtern, konnten auf dem Innovationstag Mittelstand des Bundesministeriums für Wirtschaft und Energie (BMWi) begutachtet werden. Auf der Open-AirLeistungsschau am 18. Mai in Berlin wurden 300 kleine und mittlere Unternehmen sowie Industrieforschungseinrichtungen aus dem gesamten Bundesgebiet erwartet. Sie präsentierten mehr als 200 technologiegeförderte Weltneuheiten. Bei Projekten aus den Bereichen Pflege und Rehabilitation liegt der Schwerpunkt auf einer Erhö- hung der Lebensqualität. Darunter befanden sich unter anderem ein Tanzrollator, eine Strickjacke mit integrierter Bedienfunktion und ein Mehrfunktions-Pflegesessel.

Mehrheitlich verdanken die in Berlin gezeigten Hightech-Innovationen ihre Existenz der Projektförderung im Rahmen des Zentralen Innovationsprogramms Mittelstand (ZIM) des BMWi. Das für alle Technologien und Branchen offene Programm verhilft mit einem 2017 auf 548 Mio. Euro aufgestockten Jahresbudget jährlich mehreren Tausend Entwicklungsideen aus dem Mittelstand zum Durchbruch. In den Jahren 2015 bis 2017 bewilligte das BMWi ZIM-Unterstützung in Höhe von 1,12 Mrd. Euro, etwa 92 Mio. davon gingen in die Gesundheitsindustrie. Die Hightech-Show bildet eine ideale Plattform für den Dialog zwischen Wissenschaftlern, Unternehmern, Politikern, Journalisten und interessierter Öffentlichkeit. Auch in diesem Jahr wurden auf dem weitläufigen Parkgelände der AiF Projekt GmbH in Berlin-Pankow wieder über 1.500 Besucher erwartet.

www.zim-bmwi.de 